\title{
On vanishing limits of the shear viscosity and Hall coefficients for the planar compressible Hall-MHD system
}

\author{
Xia $\mathrm{Ye}^{1}$ and Zejia Wang ${ }^{1}$ \\ ${ }^{1}$ Jiangxi Normal University
}

July 3, 2021

\begin{abstract}
This paper deals with an initial-boundary value problem of the planar compressible Hall-magnetohydrodynamic (for short, Hall-MHD) equations. For the fixed shear viscosity and Hall coefficients, it is shown that the strong solutions of Hall-MHD equations and corresponding MHD equations are global. As both the shear viscosity and the Hall coefficients tend to zero, the convergence rate for the solutions from Hall-MHD equations to MHD equations is given. The thickness of boundary layer is discussed by spatially weighted estimation and the characteristic of boundary layer is described by constructing a boundary layer function.
\end{abstract}

\section{Hosted file}

Ye-Wang.pdf available at https://authorea.com/users/423555/articles/528900-on-vanishinglimits-of-the-shear-viscosity-and-hall-coefficients-for-the-planar-compressible-hallmhd-system 